a scale from 3 to 21 using the Brief Illness Perception Questionnaire Deprivation based on postcode area was expressed as percentile ranks. For each one point increase in Anxiety, Depression and Emotional Illness Perception scores the likelihood of Poor Acceptance increased by $22 \%, 21 \%$ and $27 \%$ respectively. Greater deprivation increased the likelihood of Poor Acceptance by $2 \%$ per percentile rank.

Conclusion Predictors of Poor Acceptance may be grouped into Mood and Deprivation. Education through self-management programmes may tackle some of the problems caused by deprivation. To optimise treatment success in IBD, we would advocate further research into a mood-orientated approach using screening tools, coupled with clinical judgement and targeted psychological interventions such as Acceptance and Commitment Therapy (ACT), which is based upon Acceptance and Adjustment Theory.

Disclosure of Interest None Declared.

\section{PWE-111 ASSESSMENT AND IMPLICATIONS OF HEALTH-RELATED QUALITY OF LIFE IN A DISTRICT GENERAL COHORT OF INFLAMMATORY BOWEL DISEASE PATIENTS}

doi:10.1136/gutjnl-2013-304907.399

1,"N Swart, 'D Wellsted, ${ }^{2} \mathrm{~K}$ Lithgo, ${ }^{2 \mathrm{~T}}$ Price, ${ }^{2} \mathrm{M}$ W Johnson. ${ }^{1}$ Centre for Lifespan and Chronic IIIness Research, University of Hertfordshire, Hatfield; ${ }^{2}$ Gastroenterology, Luton \& Dunstable University Hospital, Luton, UK

Introduction Health-Related Quality of Life (HROOL) has been shown to have far-reaching implications for patient self-management, treatment efficiency and engagement with their own illness. Objectives To assess the levels of HRQOL experienced by inflammatory bowel disease (IBD) patients within the district general setting.

Methods 2400 patients with IBD in the Luton \& Dunstable catchment were invited to participate in a web-based quality of life assessment, with the option to request a paper copy. All patients were deemed eligible provided they were between 18 and 90 years of age, with no major learning difficulties or pre-existing serious mental disorders. HROOL was measured using the 10-item "short Inflammatory Bowel Disease Questionnaire" (sIBDQ; Irvine et al 1996). The sIBDQ is divided into four subscales: Systemic, Emotion, Social and Bowel. Possible scores for the sIBDQ ranged from 7 to 70 , with lower scores indicating poorer quality of life.

Results 245 patients completed the assessment (43\% male; mean age $=53, \mathrm{SD}=17$ ). $45 \%$ had Ulcerative Colitis, $45 \%$ had Crohn's Disease and $10 \%$ had an alternative form of IBD (eg. Proctitis, Lymphocytic Colitis or Collagenous Colitis). The HRQOL of patients in this study was low, and similar to that reported by Irvine et al The mean score of the sample was $48(\mathrm{CI}=46.6-49.4)$.

There are no published validated cut-off values for the SIBDO indicating a "normal" QoL. Using Huaman et al's (2010) estimated value for the IBDQ-36 (209 in the range 36-252) we assumed a cutoff of 56 in the range 7-70 to estimate a cut-off for the sIBDO. This would mean that approximately $70 \%$ of the sample had an 'abnormally' low Quality of Life. For comparison, subscale scores were divided by the number of items they contained. Mean subscale scores were: Systemic - $4.3(\mathrm{CI}=4.1-4.5)$; Emotion - $4.8(\mathrm{CI}=4.7-$ 4.9); Social - $5.4(\mathrm{CI}=5.2-5.6)$; Bowel $-4.8(\mathrm{CI}=4.6-5.0)$. A 'tailoff' effect was also noted, representing a small group of patients who reported having a significantly reduced health-related quality of life.

Conclusion This study found that Social aspects of Quality of Life were less severely affected by IBD than Emotional or Bowel-related aspects, allowing patients to maintain a relatively high level of social activity. The medical (or Bowel) aspects of the condition were perceived to have the greatest negative impact on HRQOL. However, to make significant improvements in the patients' total OOL one has to focus on improving their emotional engagement with the condition and their overall perception of the IBD and the symptoms they suffer

Disclosure of Interest None Declared.

\section{Liver}

\section{PWE-112 MATERNAL OBESITY PROMOTES OFFSPRING NON- ALCOHOLIC FATTY LIVER DISEASE (NAFLD) THROUGH DISRUPTION OF ENDOPLASMIC RETICULUM HOMEOSTASIS}

doi:10.1136/gutjnl-2013-304907.400

1, J Soeda, ${ }^{1} \mathrm{~A}$ Mouralidarane, $1,2 \mathrm{~S}$ Ray, ${ }^{1} \mathrm{R}$ Carter, ${ }^{3} \mathrm{G}$ Fusai, ${ }^{4} \mathrm{M}$ Novelli, ${ }^{5} \mathrm{~J}$ Pombo, ${ }^{5} \mathrm{~A}$ Bocioanowska, ${ }^{5} \mathrm{D}$ Sugden, ${ }^{5} \mathrm{~L}$ Poston, ${ }^{5} \mathrm{P}$ Taylor, ${ }^{1,2} \mathrm{~J}$ Oben. ${ }^{1} /$ nstitute of Liver \& Digestive Health, University College London; 'Gastroenterology, Guy's \& St Thomas' Hospitals; ${ }^{3} \mathrm{Hepatobiliary}$ Surgery \& Liver Transplantation, Royal Free Hospital; ${ }^{4}$ Department of Pathology, University College London; 'Division of Women's Health, King's College London, London, UK

Introduction We have previously shown that maternal obesity (MO) programmes offspring obesity and consequent liver disease (non-alcoholic fatty liver disease, NAFLD) but involved mechanisms are unclear. Accumulating evidence suggests endoplasmic reticulum (ER) stress induced unfolded protein response (UPR) plays a central role in the pathogenesis of steatosis and subsequent non-alcoholic steatohepatitis (NASH). However, little is known about the role of UPR in developmentally programmed NAFLD.

Methods C57BL6 mice were fed standard or obesogenic diet (OD) for 6 weeks prior to pregnancy and throughout pregnancy and lactation. Litters were weaned onto standard or OD to produce 4 groups. Animals were sacrificed at 6 months. Blood and tissue samples were collected to assess the liver phenotype and expression analysis of UPR related proteins and genes.

Results Offspring exposed to $\mathrm{MO}$ and a post-weaning OD (OffObOD) developed profound NAFLD compared to those exposed to post-partum (OffCon-OD) or the control group (OffCon-SC), as assessed by raised ALT $(p<0.001)$ and NAFLD Activity Score $(p<0.01)$. Among 3 proximal sensors of ER stress, PERK protein expression and phospho eIF-2alpha were specifically increased in OffOb-OD $(p<0.05)$. ATF6 cleavage and spliced form of XBP-1 were observed in all groups except for OffCon-SC. Phopho SAPK/ JNK, CHOP, and LC3BII protein expression were significantly increased in OffOb-OD. Furthermore, hepatocytes apoptosis as detected by TUNEL and active capase- 3 staining in OffOb-OD. These results indicate that unresolved UPR is significantly activated in OffCon-OD. However, GRP78, a major ER chaperone and central regulator for ER stress, was significantly downregulated in OffObOD. UPR induced chaperon (GRP94) and ER-associated protein degradation related gene (HERP and EDEM) were downregulated in OffCon-OB and OffOb-OD. Furthermore rhythmic expression of GRP78 and HERP were blunted in OffOb-OD.

Conclusion $\mathrm{MO}$ and a post-natal obesogenic diet profoundly disrupted ER homeostasis in offspring. Disrupted ER homeostasis may be involved in the propagation of NAFLD.

Disclosure of Interest None Declared.

\section{PWE-113 LOW RISK FOR HEPATOCELLULAR CANCER (HCC) IN HEPATITIS B VIRUS (HBV) INFECTED ASIAN MIGRANTS IMPLICATIONS FOR CANCER SURVEILLANCE}

doi:10.1136/gutjnl-2013-304907.401

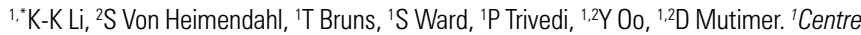
for Liver Research \& NIHR Biomedical Research Unit, University of Birmingham; 'Liver Unit, Queen Elizabeth Hospital, Birmingham, UK 